\title{
ACQUE SOTTERRANEE: RISORSA IDRICA STRATEGICA NELLA PIANURA LOMBARDA
}

\author{
ANGELO CAVALLIN,(*)(**) TULLIA BONOMI,(*) LETIZIA FUMAGALLI, $(*)$ \\ MARCO ROTIROTI(*)
}

SunTO. - L'acqua sotterranea è spesso poco considerata nei programmi gestionali perché meno "evidente" rispetto a quella superficiale, ma questa risorsa invisibile, prelevata da pozzi e sorgenti, costituisce circa il 10\% degli usi irrigui e il $95 \%$ di quelli civili (potabile, non potabile, piscicolture, etc.) dell'intera Lombardia. In provincia di Milano, l'acqua sotterranea approvvigiona la totalità dei cittadini residenti e pendolari, la quasi totalità del comparto industriale e parte di quello agricolo. Ne consegue che per questa Regione e per il comune di Milano questa risorsa è strategica e ancora di più lo sarà in futuro. La sua conservazione richiede Programmi d'Azione che ne evitino il depauperamento quantitativo e qualitativo, ma la loro efficacia dipende dalla conoscenza della risorsa idrica, ossia dell'entità delle riserve, dei suoi utilizzi, delle sue variazioni, oltre che della sua qualità. Significa quindi conoscere nel dettaglio la struttura idrogeologica e le caratteristiche delle falde idriche, ma anche disporre di una rete di monitoraggio efficiente per la misura dei livelli piezometrici e dei volumi estratti al fine di poter stimare la disponibilità idrica sotterranea e le sue variazioni. Mentre la conoscenza della struttura dei sistemi acquiferi nell'area milanese è avanzata, non lo è quella dei prelievi reali, motivo per cui la disponibilità idrica può essere stimata per via indiretta. $\mathrm{Nel}$ campo di ricerca del gruppo di Idrogeologia presso l'Università di Milano-Bicocca un settore da tempo attivo è dedicato alla parametrizzazione tridimensionale del sottosuolo, sulla base della quale è stata conseguita anche una valutazione della disponibilità idrica sotterranea. Si utilizzano a tal fine banche dati e modelli tra loro integrati in siste$\mathrm{mi}$ informativi territoriali, al fine di gestire in contemporanea una mole elevata di dati. In un'apposita banca dati idrogeologica sono stati infatti archiviati e codificati i dati stratigrafici di oltre 7000 pozzi in provincia di Milano che hanno consentito una definizione tridimensionale delle caratteristiche geometriche e idrogeologiche del sistema sotterraneo. La parametrizzazione delle proprietà idrogeologiche consente di avere un

(*) Università degli Studi di Milano-Bicocca, Dipartimento di Scienze dell'Ambiente e del Territorio e di Scienze della Terra, P.zza della Scienza 1, Milano, Italia.

${ }^{(* *)}$ E-mail: angelo.cavallin@unimib.it 
modello tridimensionale delle caratteristiche idrauliche degli acquiferi, quali la porosità efficace e la conducibilità idraulica. In particolare la conoscenza della porosità efficace ha consentito di stimare un quantitativo d'acqua potenzialmente stoccato all'interno dei sedimenti del sottosuolo, di circa 34 miliardi di $\mathrm{m}^{3}$. Tuttavia l'acqua presente nei sedimenti fini, caratterizzati da una bassa conducibilità idraulica, non è computabile come volume di acqua facilmente disponibile. La frazione d'acqua considerata sfruttabile, è quella che permea materiali medi e grossolani che presentano buona conducibilità idraulica e porosità media e medio alta $(>17 \%)$. Ne deriva che il volume idrico considerato disponibile nell'intero territorio provinciale si riduce a circa 13-15 miliardi di metri cubi d'acqua. Le variazioni di tale volume tra un anno e l'altro rappresentano il bilancio idrico netto del sistema idrogeologico, positivo o negativo in funzione della relazione tra le entrate nel sistema, denominate anche "ricarica", (precipitazioni efficaci, irrigazioni efficaci, perdite da canali e fognature, alimentazione da fiumi, etc.) e le uscite (prelievi, fontanili, drenaggi da fiumi, etc.). La conoscenza di questi fattori che incidono sul bilancio permetterebbe di definire un livello minimo sostenibile della risorsa e, di conseguenza, di indicare i quantitativi che ragionevolmente potrebbero essere sfruttati a fronte di una quantità che sempre e comunque deve rimanere a costituire una riserva strategica. Inoltre fenomeni di contaminazione presenti nell'area limitano ulteriormente il volume di acqua sfruttabile e richiedono costi elevati per opere di disinquinamento e impianti di depurazione. La conoscenza quali-quantitativa di questa risorsa è quindi uno strumento utile per la sua gestione, che potrebbe anche fornire indicazioni nella gestione di potenziali incrementi di consumi, anche sporadici, come atteso per esempio nel caso dell'Expo 2015 di Milano. Infatti la possibilità di stimare i volumi idrici disponibili consente di effettuare simulazioni al fine di predire le variazioni indotte sulla risorsa da impatti di origine diversa, naturali e/o antropici, all'interno di scenari logici e plausibili.

$$
* * *
$$

ABSTRACT. - A proper understanding of the water cycle, and in particular of the distribution and availability of groundwater will enable to evaluate their sustainability and manage them especially in critical situations which in the future will be related to climatic change with high temperature conditions, in which more consumable water is needed. Water use in Lombardy region is an economic opportunity and related consumption are described. To satisfy water uses it is necessary to develop an integrated system for a rational management in which groundwater have an important role. In DISAT researches to evaluate groundwater resources are developed by long time. Mathematical models applied to hydrogeology are used to verify and simulate real systems and to predict future scenarios. The methodology use an integrate system from a database of wells to software which permit to reconstruct the 3D geometry of subsoil and parameterize it according to the texture distribution of the subsoil to evaluate the water storage and its availability. According to these application and coupling models it will be possible, in the future, simulate the effects of critical situations related to groundwater and to identify the best solutions. 


\section{INTRODUZIONE}

L'acqua sotterranea è poco considerata nei programmi gestionali perché risulta "meno evidente" rispetto a quella superficiale, ma questa risorsa invisibile, prelevata da pozzi e sorgenti, costituisce circa il 10\% degli usi irrigui e il $95 \%$ di quelli civili (potabile, non potabile, piscicolture, etc.) dell'intera Lombardia [1] e, per la provincia di Milano, l'acqua sotterranea approvvigiona la totalità dei cittadini residenti e pendolari, la quasi totalità del comparto industriale e parte di quello agricolo. Ne consegue che per questa Regione e per il suo capoluogo, questa risorsa è, ancora di più sarà, la più strategica.

In Lombardia le acque rappresentano una straordinaria opportunità non solo per gli aspetti ambientali ma soprattutto per quelli socioeconomici. Infatti l'uso delle acque per l'agricoltura è stato e continua ad essere il motore principale dello sviluppo agricolo della pianura, mentre quello per la produzione di energia ha permesso un elevato sviluppo industriale e continua a fornire un notevole contributo in termini di apporti energetici da fonti rinnovabili. Inoltre la diffusa disponibilità idrica costituisce una risorsa essenziale per gli insediamenti industriale e i processi produttivi. Anche la grande importanza che hanno le attività connesse con il turismo sono legate alla presenza della acque ed in particolare di laghi e di ambienti naturali di pregio ad elevata attrattiva turistica.

\section{L'USO DELLE RISORSE IDRICHE IN LOMBARDIA}

Il PTUA [1] descrive la Lombardia come una regione caratterizzata da un apporto idrico da precipitazione elevato, stimato in circa 27 miliardi di mªnno, su un territorio di $23.856 \mathrm{~km}^{2}$, di cui $42 \%$ montano, $13 \%$ collinare e $45 \%$. La media delle precipitazioni è di circa $1.000 \mathrm{~mm} / \mathrm{anno}$, con valori massimi di circa $2.000 \mathrm{~mm} /$ anno nella zona prealpina occidentale e minimi di circa $600 \mathrm{~mm} / \mathrm{anno}$ nella bassa pianura.

Il territorio è caratterizzato da una densità abitativa tra le più elevate in Italia e in Europa che, nel settore centrale pedemontano e di pianura, è simile a quella delle aree dipartimentali di Parigi e Bruxelles. Qui si sono infatti concentrate attività ad alto impatto sia sul territorio sia in particolare sulle risorse idriche. La superficie agraria utilizzata è pari circa alla metà del territorio regionale, con netta prevalenza dei 
seminativi ed è molto diffuso l'allevamento di bovini, suini e avicoli.

Le necessità idriche sono di tipo civile, industriale, irriguo, ultimamente anche geotermico, cui vanno aggiunti anche quelli di carattere ambientale e turistico-ricreativo come: pesca, balneazione, navigazione.

Un censimento degli utilizzi idrici in Lombardia [1] valuta che le portate di acqua, superficiale e sotterranea, concesse in Lombardia per i diversi usi (civile potabile e non potabile, cioè igienico, antincendio, zootecnico, industriale, irriguo, piscicoltura, produzione di energia) ammontano complessivamente a circa 130 miliardi $\mathrm{di}^{3} /$ anno, vale a dire 5 volte di più dell'afflusso meteorico annuo sul territorio lombardo. Tuttavia, se dall'analisi viene sottratto l'utilizzo per la produzione di energia, la portata regionale concessa ammonta a circa 37 miliardi $\mathrm{m}^{3} /$ anno, di cui più dell' $80 \%$ destinati all'uso irriguo, il $12 \%$ al civile e il $5 \%$ all'industriale (depurato dell'uso per raffreddamento centrali) (Fig. 1).
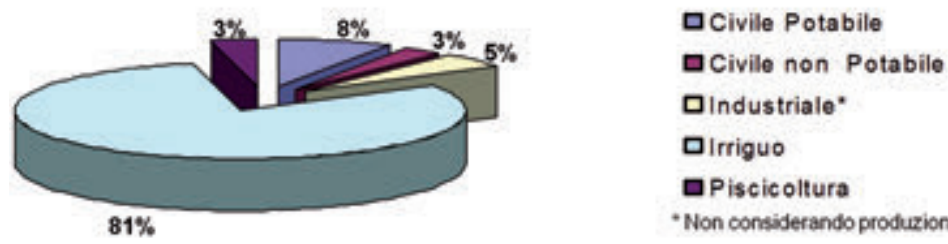

* Non considerando produzione energia

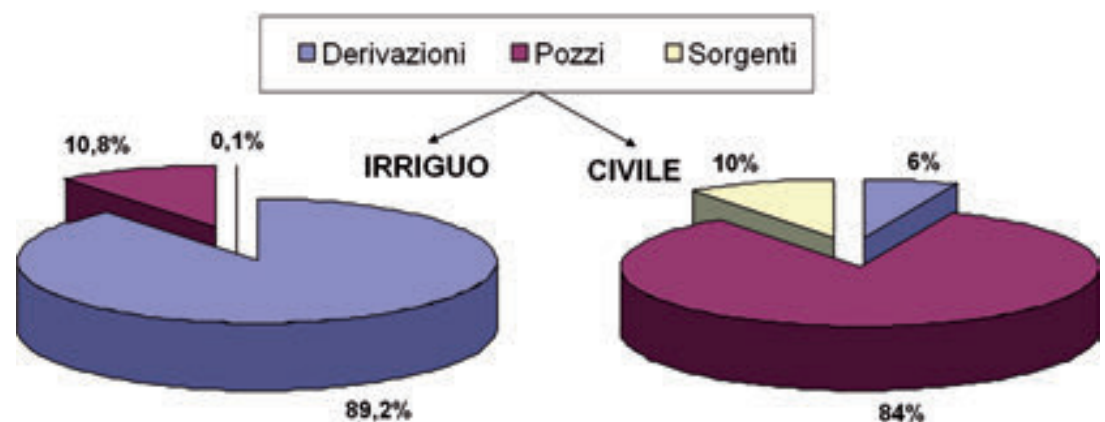

Fig. 1. Usi delle acque in Lombardia escluso quello per la produzione di energia.

(Da [1] modificato).

Le acque derivate a uso irriguo, che approvvigionano circa 700.000 ha di superficie agricola, provengono prevalentemente da acque superficiali $(89 \%)$. Il fabbisogno residuo è fornito dalla falda (11\%). Il prelievo da acque superficiali avviene attraverso un centinaio di grandi derivazioni, e la distribuzione alle aree irrigate è effettuata mediante una fitta rete 
di canali. Si stima che almeno il 50\% dell'acqua fornita al terreno non sia utilizzata dalle colture, ma percoli nelle falde sotterranee e rifluisca nel reticolo scolante. L'irrigazione sottrae rilevanti quantità d'acqua in brevi periodi, senza poi restituirla, al reticolo superficiale [1].

L'approvvigionamento idrico a scopo potabile usa prevalentemente acque sotterranee, con un numero di captazioni totale tra pozzi e sorgenti di circa 9.000 a fronte di circa 30 derivazioni da acque superficiali. In termini di volumi il contributo delle acque superficiali è di circa il 5\%. Tipicamente utilizzati per l'approvvigionamento potabile sono anche i grandi bacini lacustri e, nelle aree di montagna, le sorgenti. Nell'area di pianura il prelievo avviene essenzialmente da acque sotterranee, con circa 6.000 pozzi, che raggiungono talvolta profondità ragguardevoli (200-300 m). Come prevedibile, le zone di maggior sfruttamento per usi civili si concentrano nell'area più densamente popolata della regione rappresentata dal nord milanese, idrograficamente posto tra l'Olona e il Lambro [1].

\section{LE CRITICITÀ IDRICHE}

La domanda di risorse idriche per i vari usi, con concessioni per 130 miliardi $\mathrm{di}^{3} /$ anno, supera di molto la disponibilità, con precipitazioni per 27 miliardi di $\mathrm{m}^{3} /$ anno, per cui si viene a creare una condizione di grave carenza idrica. Anche tenendo conto che le concessioni di acque per uso idroelettrico non portano a un reale consumo delle risorse idriche, in quanto riutilizzate più volte lungo le aste dei corsi d'acqua, e che le riserve idriche regionali (laghi:120 miliardi di $\mathrm{m}^{3}$, ghiacciai: 4 miliardi di $\mathrm{m}^{3}$, falde sotterranee: oltre 100 miliardi $\mathrm{di}^{3}$ ) sono tali da far fronte a particolari situazioni di emergenza, esiste un reale problema di gestione delle risorse idriche regionali, anche a livello di bacino padano.

Se si considerano inoltre le valutazioni fatte dalla ARPA Emilia Romagna processando i dati relativi alle precipitazioni e temperature di tutte la Arpa della Pianura padana a partire dagli anni '70 [2] si osserva quanto segue:

- precipitazioni in calo e regime naturale alterato, piogge di maggiore intensità, ma con minore frequenza, soprattutto nel periodo invernale primaverile; nel complesso deficitarie rispetto alle captazioni nella stagione estiva e idraulicamente pericolose in quella autunnale; 
- temperature crescenti;

- deflussi in chiusura di bacino diminuiti del 20-25\% in seguito al calo degli afflussi, particolarmente in estate, e all'aumento della evaporazione per incremento della temperatura;

- precipitazioni nevose e volume dei ghiacciai alpini in diminuzione;

- previsione di incrementi di temperatura futuri simili agli attuali, per complessivi $2-3^{\circ} \mathrm{C}$ a fine secolo, e riduzione lieve della precipitazione in tutte le stagioni salvo in autunno, nel quale tuttavia ne aumenta la variabilità.

In effetti situazioni critiche per quanto concerne la disponibilità di acqua si sono già verificate in passato, nel 2003 e nel 2006 colpendo in particolare le produzioni agricole.

Per affrontare tali criticità si deve prevedere pertanto un utilizzo razionale, consapevole e sostenibile di tale risorsa in accordo con la Direttiva Quadro n. 2000/60/CE del 23 ottobre 2000 [3], che assume come oggetto di tutela non solo l'acqua, ma tutto l'ambiente acquatico e territoriale circostante, individuando come unità di riferimento per la pianificazione e la gestione delle risorse idriche il bacino idrografico. Sicuramente un risorsa strategica in quest'ottica gestionale è rappresentata dalle acque sotterranee, in particolare nelle aree di pianura.

\section{L'UTILIZZO DELLE RISORSE IDRICHE SOTTERRANEE}

Un importante effetto dell'utilizzo delle acque sotterranee è rappresentato dalle variazioni spazio-temporali della superficie della falda in funzione delle diverse caratteristiche geologiche e idrogeologiche e dell'uso del territorio. Infatti il livello della falda è l'effetto sinergico delle componenti delle voci di bilancio in entrata (precipitazioni, irrigazioni, infiltrazione delle acque superficiali, apporti dalle falde da monte) e in uscita (prelievi da pozzi, fontanili, drenaggio da corsi d'acqua) in funzione delle geometrie e delle caratteristiche idrogeologiche dei corpi che costituiscono il sottosuolo (Fig. 2).

Nella città di Milano, dove l'approvvigionamento idrico avviene per emungimento dalle acque sotterranee tramite pozzi, si è riscontrato in un graduale abbassamento della falda fino alla fine degli anni ' 70 per poi assistere al suo progressivo innalzamento negli anni successivi (Fig. 3). Questo è il risultato di un squilibrio delle voci di bilancio: 
quando le componenti di uscita sono maggiori di quelle di entrata l'effetto è un abbassamento, viceversa quando quelle di entrata sono maggiori di quelle in uscita si osserva un innalzamento [5].

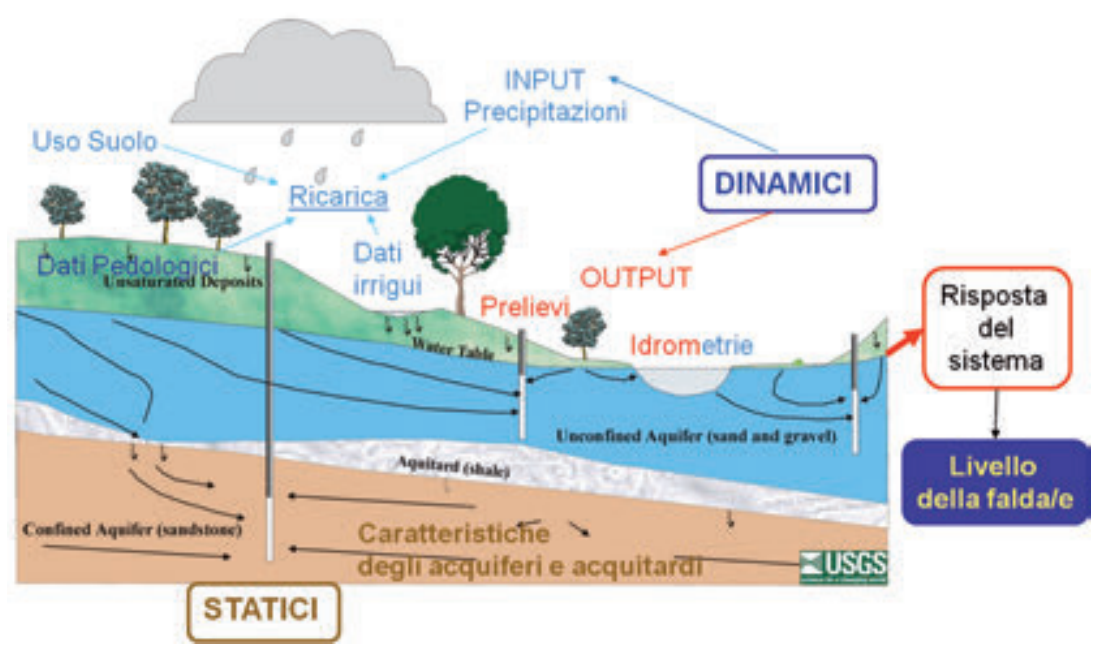

Fig. 2. Ciclo delle acque sotterranee. (Da [4] modificato).

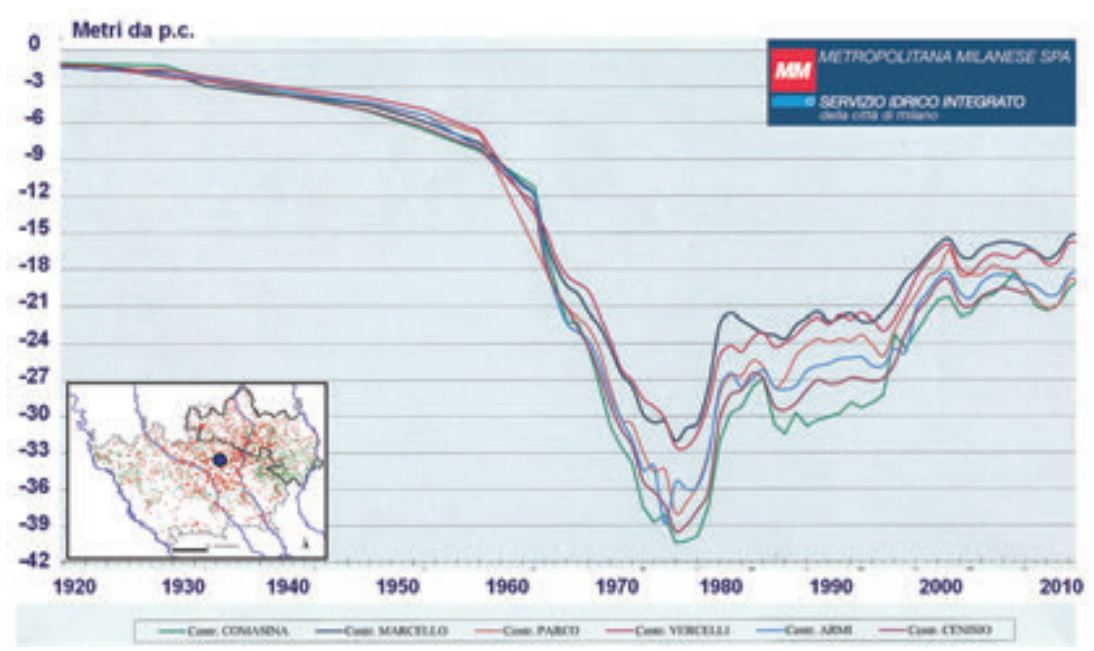

Fig. 3. Andamento piezometrico a Milano dal 1920 al 2010. 
Nell'area milanese la risposta del sistema falda è diversa nelle zone irrigue, non irrigue e urbane (Fig. 4) e viene di seguito analizzata sulla base di elaborazioni effettuate sui dati piezometrici provinciali del periodo 1975-2006.

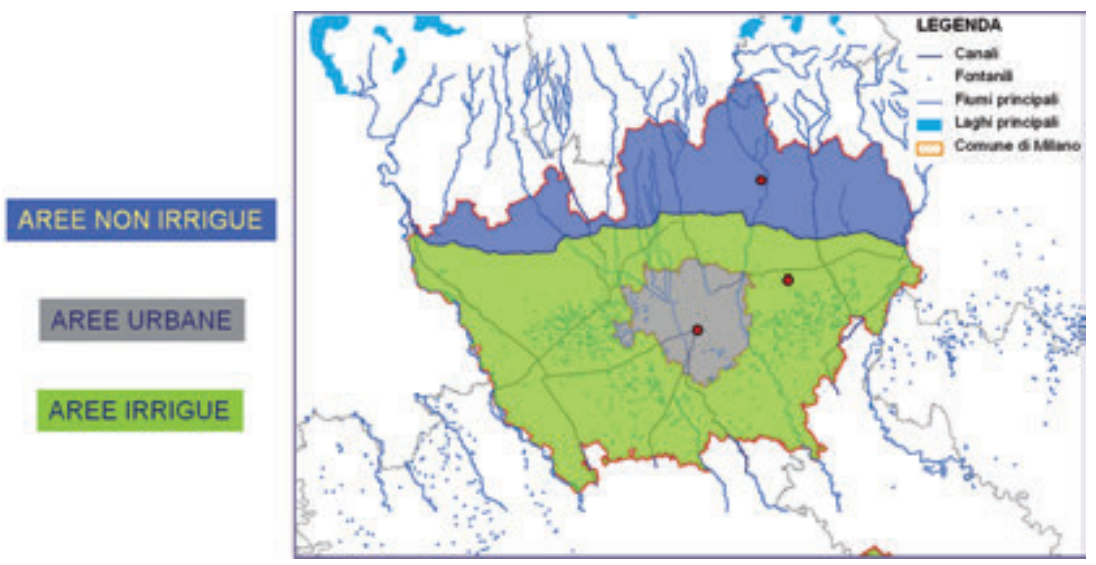

Fig. 4. Zonazione delle aree irrigue, non irrigue e urbane dell'area milanese s.l., e ubicazione dei punti di misura della falda riportati nelle Figg. 5, 6 e 7.

Nella zona più a nord, senza irrigazioni, l'andamento tipico della falda è riportato nella Fig. 5 [6] in cui la variazione della piezometria a Muggiò è comparata con l'indice SPI12 (Standard Precipitation Index a 12 mesi) a Monza. Il confronto indica che periodi prolungati di precipitazioni superiori o inferiori alle medie hanno un riscontro evidente sull'andamento delle acque sotterranee, ove non vi siano altre voci di bilancio nettamente prevalenti. Tale corrispondenza presenza uno sfasamento temporale di almeno un anno. Ove le misurazioni piezometriche non fossero state temporalmente continue, con frequenza almeno mensile, non è stato possibile fare alcuna valutazione.

Le variazioni piezometriche nelle zone con irrigazioni sono riportate nella Fig. 6. Il trend annuale evidenzia la forte stagionalità del contributo irriguo. Lo sfasamento di qualche mese di ritardo è dovuto al tempo di utilizzo dell'acqua da parte delle coltivazioni, alla tipologia di suolo presente e al tempo di transito e percolazione nella zona non satura. Il trend pluriannuale del livello della falda è invece influenzato, anche in questo caso, dalle precipitazioni.

Nella aree fortemente antropizzate la risposta della falda ha un 
andamento rappresentato nella Fig. 3 (1920-2010) e nella Fig. 7 (19512010) [6].

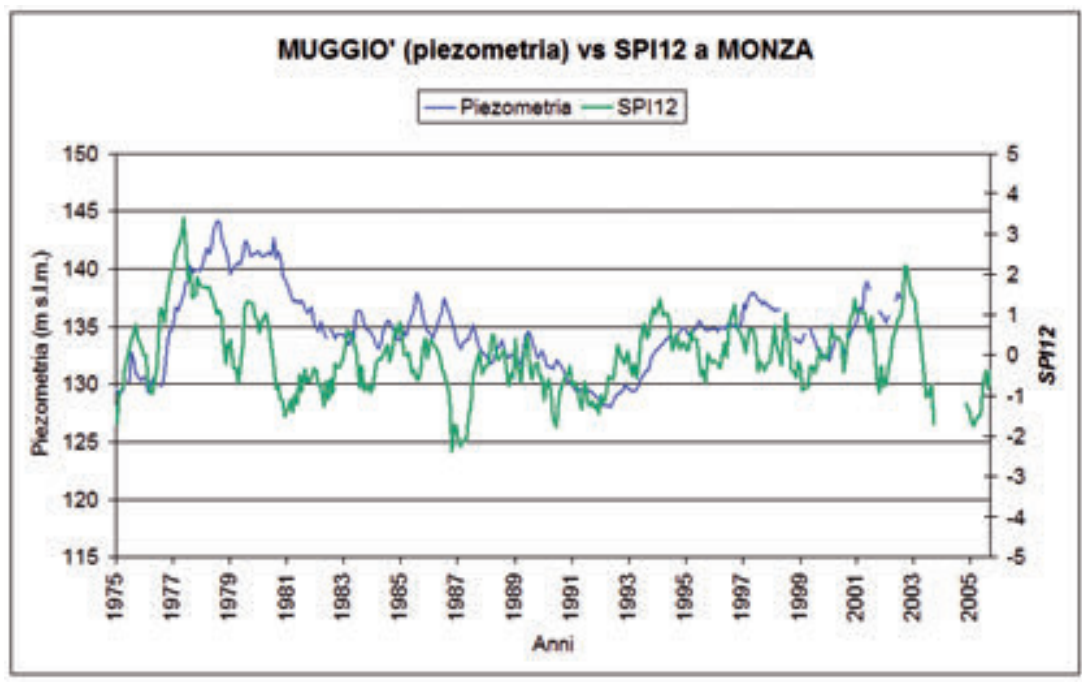

Fig. 5. Andamento della falda in una area non irrigua [6].

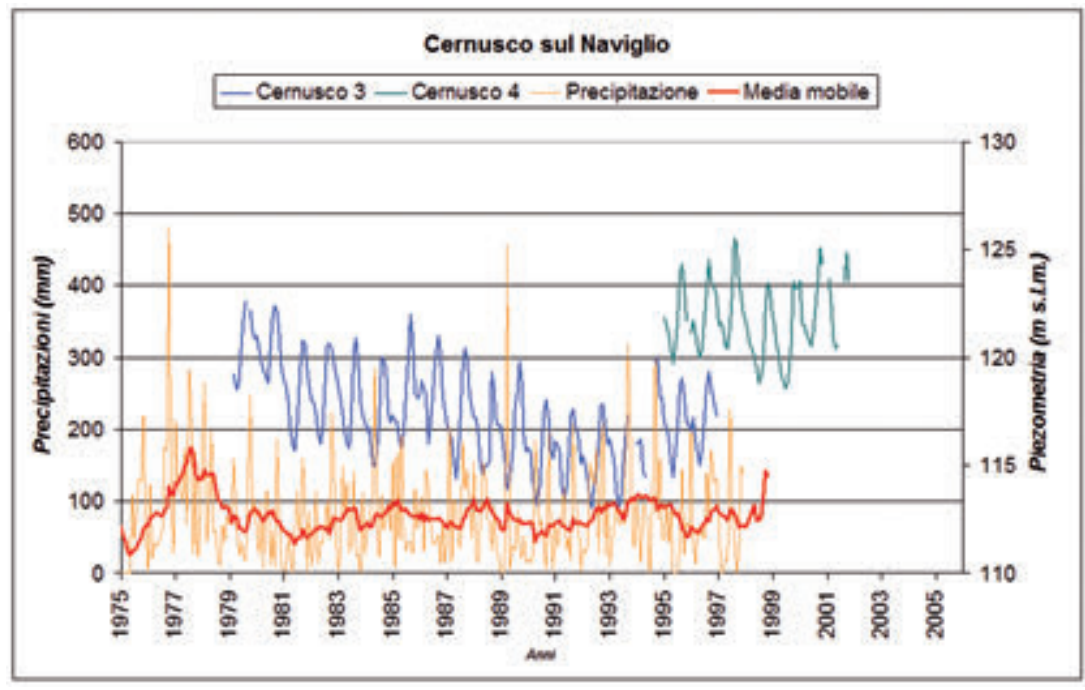

Fig. 6. Andamento della falda in un'area irrigua [6]. 


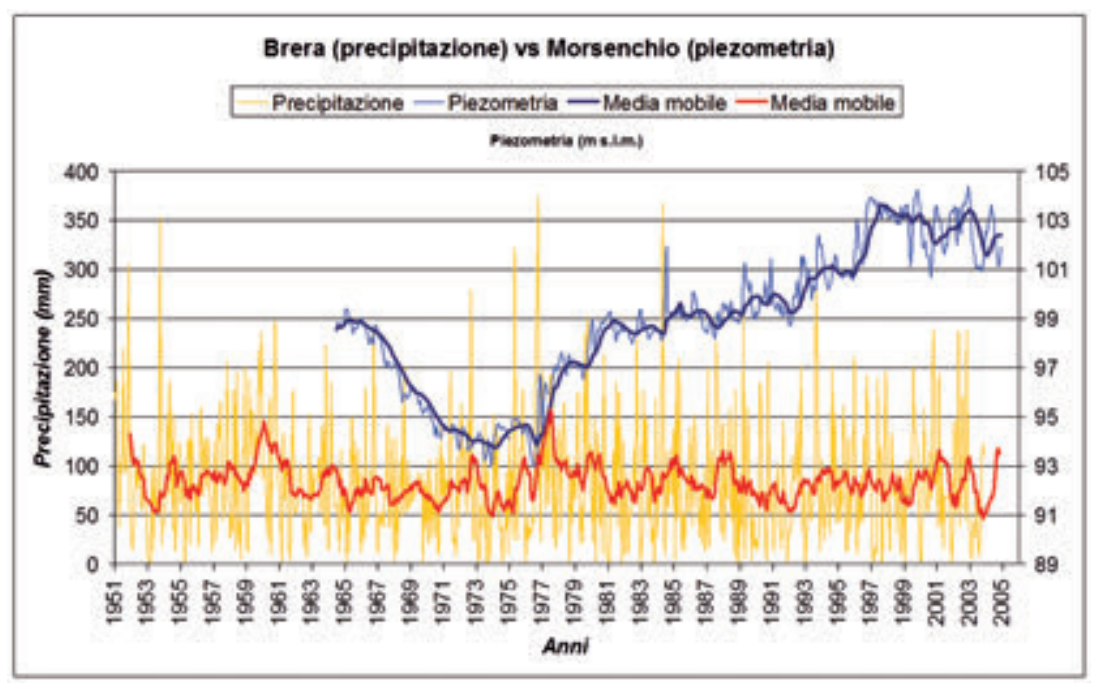

Fig. 7. Andamento della falda in un'area fortemente antropizzata (Milano) [6].

Si può osservare in entrambe come il trend della falda su lungo periodo non risente di variazioni climatiche significative in quanto l'andamento piezometrico crescente è fortemente influenzato da una drastica riduzione degli emungimenti dovuto a una riduzione della popolazione residente ma soprattutto alla notevole riduzione delle attività industriali idroesigenti come quelle del settore nord di Milano (Pirelli, Falk, ecc.).

Per poter valutare e gestire le problematiche connesse con la disponibilità idrica, il cui indicatore è rappresentato dall'andamento del livello della falda, è necessario sviluppare programmi d'azione per evitarne il depauperamento quantitativo e qualitativo. Tali programmi, per essere efficaci, richiedono una approfondita conoscenza della risorsa idrica stessa, ossia dell'entità delle riserve, dei suoi utilizzi e delle sue variazioni, oltre che della sua qualità.

\section{RicostruZioni 3D DEL SOTTOSUOLO}

Ricerche mirate a conseguire una valutazione della disponibilità idrica sotterranea vengono da tempo svolte dal gruppo di Idrogeologia dell'Università di Milano-Bicocca mediante lo sviluppo di una metodica che consente di ottenere una parametrizzazione tridimensionale del 
sottosuolo. Tale metodica comprende un uso integrato di banche dati e modelli, in sistemi informativi territoriali, che consentono una gestione contemporanea di una mole elevata di dati. In un'apposita banca dati idrogeologica sono stati infatti archiviati e codificati i dati stratigrafici di oltre 35.000 pozzi prevalentemente della Regione Lombardia di cui oltre 7600 pozzi per la provincia di Milano (Fig. 8) la cui elaborazione consente di definire tridimensionalmente le caratteristiche geometriche e idrogeologiche (conducibilità e porosità) del sistema sotterraneo.

Nello specifico la metodologia integra banche dati per pozzi per acqua TANGRAM [7,8], GIS (Geographic Information System) e software 3D GOCAD [9].

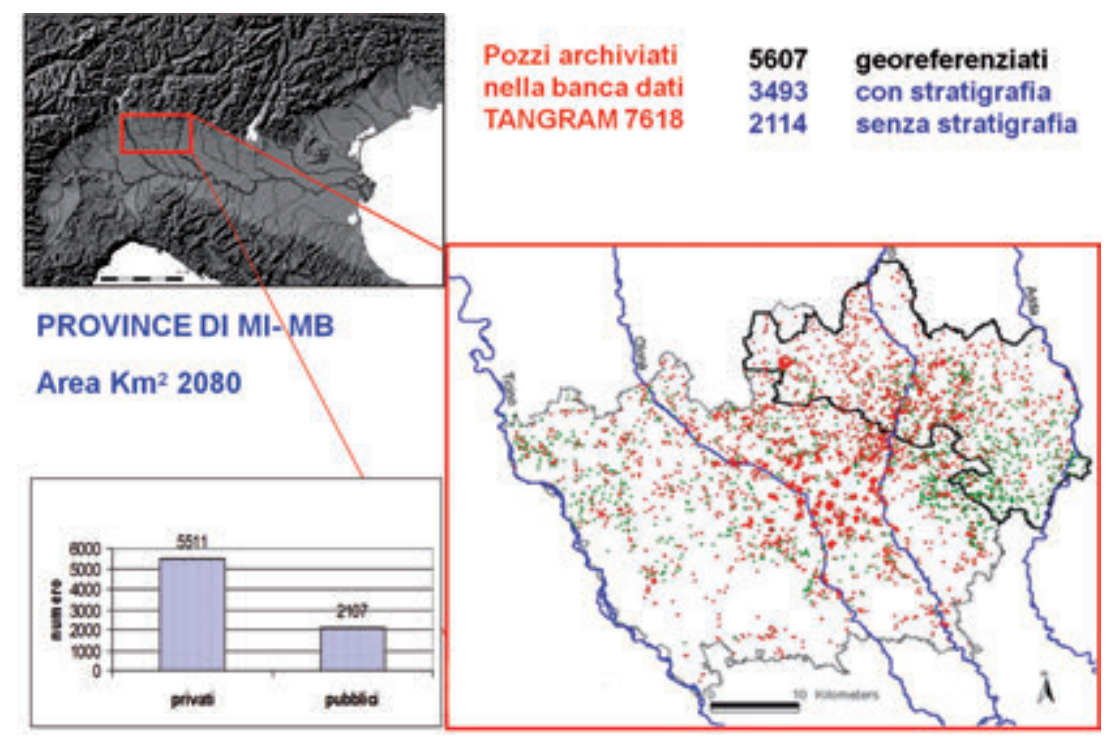

Fig. 8. Localizzazione e tabella sinottica dei pozzi nelle provincie di Milano e Monza-Brianza.

Le fasi per la ricostruzione delle caratteristiche fisiche del sistema prevedono: 1 - archiviazione delle informazioni idrogeologiche dei pozzi in TANGRAM nella quale i dati stratigrafici dei pozzi sono codificati e georeferenziati; 2 - estrazione dalla banca dati in coordinate spaziali, delle percentuali dei termini tessiturali e delle caratteristiche idrogeologiche di permeabilità e porosità attribuite ad ogni singolo livello stratigrafico attraversato dai pozzi; 3 - elaborazione degli stessi secondo intervalli normaliz- 
zati, identificati sulla base della scala di dettaglio prescelta; 4 - ricostruzio$n e 3 \mathrm{D}$ in GOCAD delle geometrie di riferimento del sistema idrogeologico in esame; 5 - conversione dei dati archiviati nella banca dati in punti XYZ e creazione di una griglia tridimensionale (Sgrid) in GOCAD, delimitata dalle superfici di controllo del sistema ed interpolazione tridimensionale con l'uso del DSI, (Discrete Smooth Interpolator) e/o di tecniche kriging; 6 - ricostruzione $3 \mathrm{D}$ delle caratteristiche tessiturali ed idrogeologiche del sottosuolo, per la profondità voluta e con intervalli a scelta.

Mediante l'uso di GOCAD (Geologic Object Computer Aided Design) [9] possono essere ricostruite le geometrie tridimensionali di riferimento del sistema idrogeologico in esame (per esempio superficie topografica, base degli acquiferi), rispetto alle quali posizionare i pozzi archiviati nella banca dati.

Dalla banca dati vengono selezionati, per ogni pozzo georeferenziato, i valori percentuali dei termini grossolani, medi e fini, per intervalli di profondità a scelta e successivamente elaborati nel software GOCAD come PointsSet, unitamente ai dati relativi alle superfici di riferimento del sistema (superficie topografica, base del primo acquifero). I punti XYZ ricavati dalla banca dati vengono interpolati tridimensionalmente, secondo la griglia predefinita, con l'uso di tecniche geostatistiche (kriging). Con questi elementi è stata elaborata una griglia tridimensionale sull'intera provincia di Milano costituita da celle di $250 \mathrm{~m} * 250 \mathrm{~m}$ di lato e circa $3 \mathrm{~m}$ di spessore. La profondità totale considerata è circa $100 \mathrm{~m}$, variabile in funzione delle superfici. L'attribuzione dei valori percentuali delle tessiture dei singoli pozzi, a tutte le celle che costituiscono l'SGrid, ha consentito di elaborare tridimensionalmente le distribuzioni delle caratteristiche tessiturali e di interpretarle in dettaglio.

Tali elaborazioni consentono di calcolare la distribuzione percentuale dei termini grossolani sull'intero volume di sottosuolo che va dal piano campagna alla base del primo acquifero [10]. E' possibile visualizzare sezioni lungo le tre direzioni $(\mathrm{x}, \mathrm{y}, \mathrm{z})$ per tutte le classi granulometriche considerate (grossolane, medie e fini) e individuare i principali livelli permeabili del sottosuolo provinciale e il loro andamento spaziale. La rappresentazione a colori della presenza percentuale di ogni singola frazione tessiturale ne evidenzia la distribuzione spaziale (Fig. 9) [11].

L'interpretazione delle presenze relative dei termini tessiturali consente invece di ottenere una parametrizzazione del volume studiato ottenendo le distribuzioni tridimensionali della conducibilità idraulica e della porosità efficace (Fig. 10) [11]. 


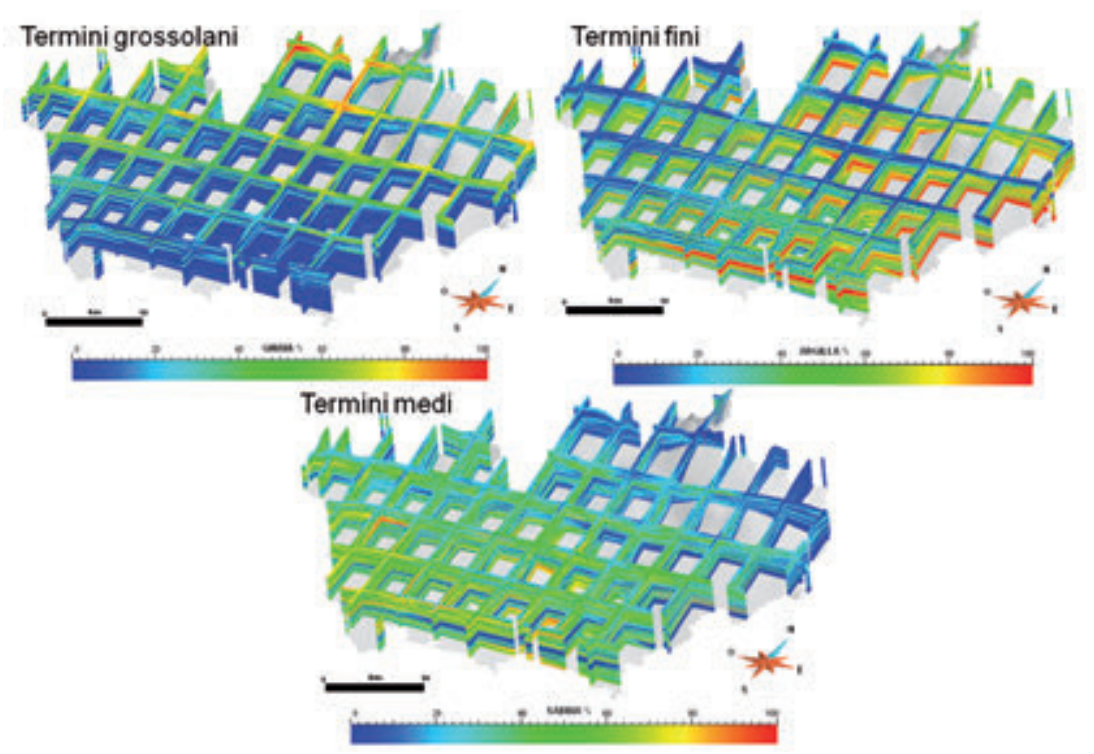

Fig. 9. Distribuzione delle caratteristiche tessiturali nel sottosuolo dell'area milanese [11].
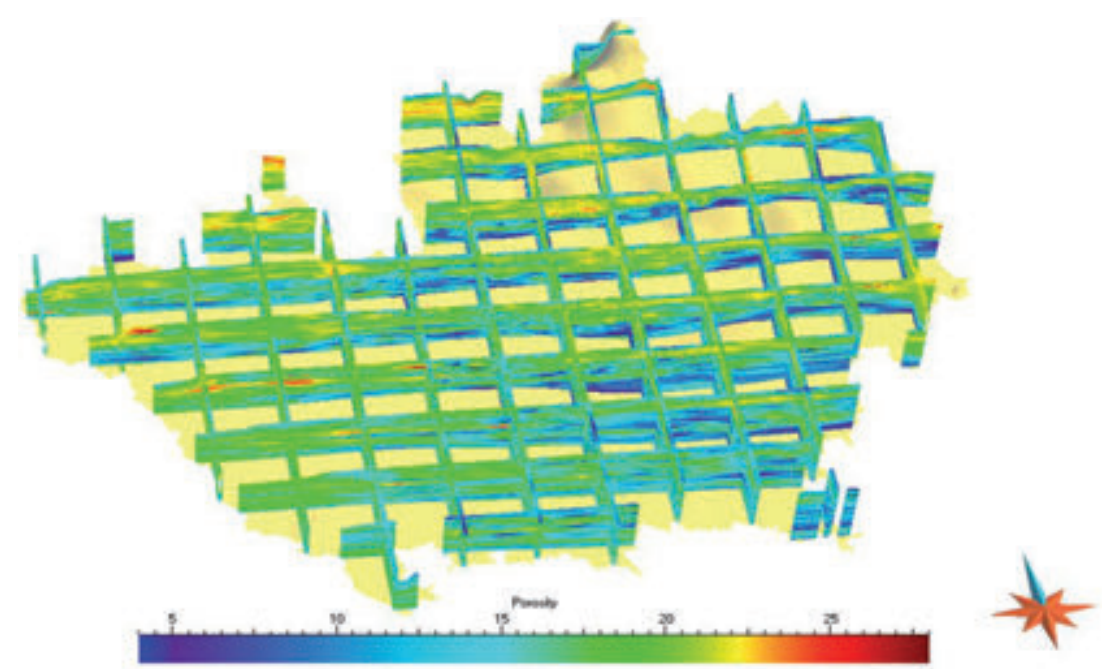

Fig. 10. Distribuzione della porosità efficace nel sottosuolo dell'area milanese [11]. 


\section{VALUTAZIONE DELLE RISORSE IDRICHE SOTTERRANEE DISPONIBILI}

Nel caso del comune di Milano il volume investigato è pari a oltre 217 miliardi di $\mathrm{m}^{3}$ di terreno, del quale una parte è satura ed una parte non satura. Tale distinzione varia dinamicamente con il variare della superficie piezometrica.

Quindi il calcolo dei volumi d'acqua immagazzinati nel sottosuolo richiede una ricostruzione dell'andamento piezometrico spazio-temporale, per cui i dati relativi al monitoraggio del livello della falda sono fondamentali per poter valutare la disponibilità delle risorse idriche. Nel caso di elaborazioni quantitative della riserva di seguito presentato sono stati utilizzati i dati del monitoraggio effettuato da1972 al 2005 dalla Provincia di Milano, che indicano una evoluzione temporale del livello piezometrico assai dinamica nel tempo (Figg. 3 e 7).

Si osservi che il variare del livello piezometrico cambia le proporzioni tra zona satura e non satura e che, essendo queste caratterizzate da differenti classi granulometriche e quindi da differenti classi di porosità efficace, produce variazioni dei volumi d'acqua presenti nel sottosuolo.

Nella Fig. 11 sono riportate le variazioni del volume d'acqua immagazzinato nel sottosuolo provinciale in relazione alle variazioni piezometriche e alla ricostruzione stratigrafica tridimensionale, per alcuni anni dal $1979 \mathrm{al} 2005$. Esse sono ottenute attraverso la parametrizzazione del sottosuolo e la definizione della porosità efficace. In tale periodo, in accordo a quanto visto con l'evoluzione temporale in un singolo pozzo, si osserva una minima disponibilità di acqua nel 1992 (minimo piezometrico, 32,9 miliardi di mc) e una massima disponibilità di acqua nel 1980 (massimo relativo con 34,3 miliardi di mc effetto di elevate precipitazioni, Fig. 11).

Si osservi tuttavia che delle riserve sopra citate, una parte interessa sedimenti a bassa porosità e bassa conducibilità idraulica, per cui non può essere considerata disponibile. La riserva disponibile è quella che permea corpi costituiti da depositi tessiturali medi e grossolani con porosità media e medio alta (superiore al 17\%). Ne consegue che, dei circa 34 miliardi di $\mathrm{m}^{3} \mathrm{~d}$ 'acqua sopra indicati, solo circa 13-15 miliardi di $\mathrm{m}^{3}$ risultano disponibili nell'intero territorio provinciale (Fig. 12).

Le variazioni della riserva tra un anno ed il successivo possono essere considerate il bilancio idrico netto del sistema idrogeologico, pari alla differenza tra la ricarica del sistema (precipitazioni efficaci, irrigazioni efficaci, perdite di canali e fognature, alimentazione da fiumi, etc.) e l'uscita (prelievi, fontanili, drenaggi da fiumi, etc.). La conoscenza di ognu- 
no di tali fattori permetterebbe di definire un livello minimo sostenibile della falda e, di conseguenza, il quantitativo che sarebbe ragionevole pensare di poter sfruttare e quello che dovrebbe sempre e comunque essere non sfruttato in quanto costituisce una riserva strategica.

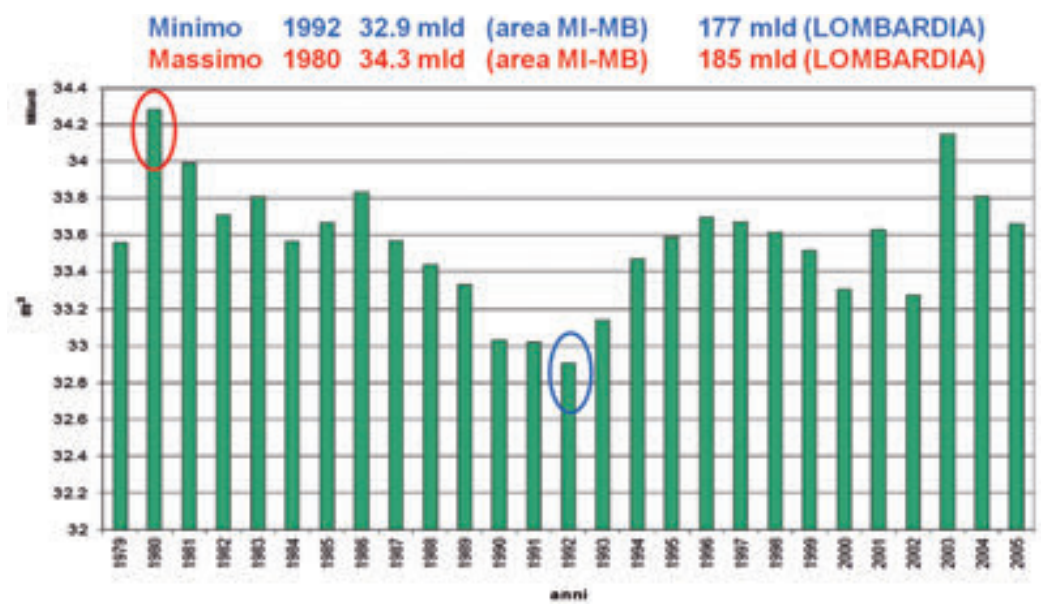

Fig. 11. Volume totale di acqua immagazzinata nell'acquifero della provincia milanese.

(Da [11] modificato).

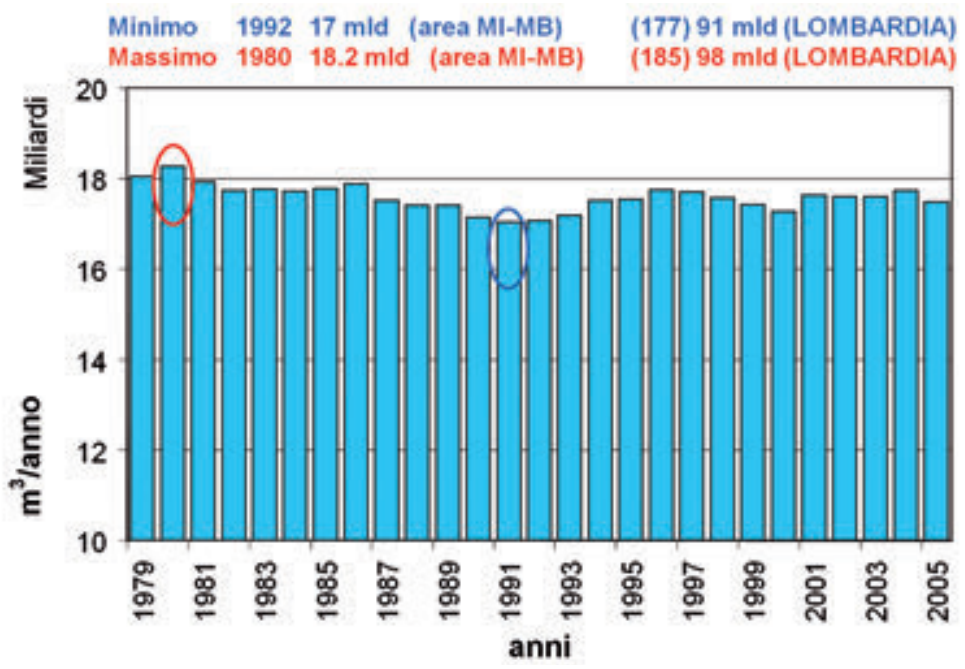

Fig. 12. Volume di acqua disponibile nell'acquifero della pianura milanese. 


\section{CONSIDERAZIONI FINALI}

Le acque sotterranee hanno tempi di risposta lunghi, per cui un evento meteorico, che sia siccitoso o intenso, di durata giornaliera, mensile o anche annuale, non riesce a riflettersi sulle acque sotterranee in modo significativo. Quando invece la durata è pluriennale, allora anche il sistema sotterraneo ne risente, positivamente o negativamente, con significative delle variazioni dell'entità dell'immagazzinamento, evidenziate dall'andamento della superficie piezometrica.

Per valutare tali variazioni necessario quindi disporre di reti di monitoraggio la cui frequenza di misura sia però almeno mensile.

La conoscenza quali-quantitativa delle riserve sotterranee è lo strumento fondamentale per la loro gestione. L'approccio utilizzato per stimare i volumi idrici disponibili appare essere una via affidabile per arrivare a simulare, mediante modelli, l'impatto che potrebbero avere sulla risorsa idrica sotterranea variazioni delle entrate e/o delle uscite, legate sia a cause naturali che antropiche. Tra gli scenari di simulazione plausibili possibili, si potrebbero indicare, per esempio, sia le variazioni climatiche sia l'incremento dei consumi idrici che potrebbe verificarsi a Milano in concomitanza di particolari eventi, cui partecipano milioni di persone per vari mesi, quali l'Expo 2015.

\section{BIBLIOGRAFIA}

[1] Regione Lombardia, (2006). Usi e tutela delle acque in Lombardia. Linee strategiche, pianificazione e regole per un utilizzo razionale e sostenibile della risorsa idrica, Regione Lombardia,. Milano, 2006.

[2] Tibaldi S., (2007). Il cambiamento climatico nel bacino del Po: variabilità e trend. Atti della conferenza "Effetti dei cambiamenti climatici sul bacino del Po", 16 luglio 2007, Conferenza Cambiamenti Climatici, Parma aprile 2007.

[3] Commisione Europea, (2000), Direttiva Quadro sulle Acque, 2000/60/CE, 2000, Brussel.

[4] United States Geological Survey, sito: http://ga.water.usgs.gov/edu/watercycle italian.html

[5] Bonomi T., Cavallin A., (2007), "La struttura idrogeologia e le variazioni piezometriche: elementi critici nella gestione delle acque sotterranee", Giornale di Geologia Applicata, 2007, 5 (2007), pp. 125-134.

[6] Bonomi, T., Canepa, P., Del Rosso, F., \& Rossetti, A., (2008). Relazioni temporali pluridecennali di dati pluviometrici, idrologici e piezometrici nella pianura lombarda tra Ticino e Oglio. Giornale di geologia applicata, 2008, 9(2), 227-248. 
[7] Bonomi T., Cavallin A., De Amicis M., (1995), "Un database per pozzi: Tangram", Quaderni Geologia Applicata, 1995, suppl. n. 31/95, 3.461-3.465.

[8] Bonomi, T., Fumagalli, M.L., Rotiroti, M., Bellani, A., \& Cavallin, A. (2014)., Banca dati idrogeologica TANGRAMC: strumento per elaborazioni quantitative di dati per la valutazione delle acque sotterranee. Acque sotterranee, 2014, $3(2 / 136), 35-45$.

[9] Gocad Suite, (2005). Earth Decision, 2005.

[10] Bonomi T., (2009), "Database development and 3D modeling of textural variations in Heterogeneous, unconsolidated aquifer media: application to the Milan plain”, Computers \& Geosciences, 2009, 35, 134-145.

[11] Bonomi, T., Del Rosso, F., Fumagalli, M.L., \& Canepa, P., (2010). Assessment of groundwater availability in the Milan Province aquifers,. Mmemorie descrittive della Ccarta Ggeologica d'Iitalia, 2010, 90, 31-40. 